\title{
Cytomegalovirus infections in lung and hematopoietic cell transplant recipients in the Organ Transplant Infection Prevention and Detection Study: A multi-year, multicenter prospective cohort study
}

\author{
Robin K. Avery ${ }^{1}$ (D) | Fernanda P. Silveira ${ }^{2}$ | Kaitlin Benedict $^{3}$ | Angela A. Cleveland $^{3}$ | \\ Carol A. Kauffman ${ }^{4}$ | Mindy G. Schuster ${ }^{5}$ | Erik R. Dubberke ${ }^{6}$ | Shahid Husain ${ }^{7}$ | \\ David L. Paterson ${ }^{8}$ | Tom Chiller ${ }^{3}$ | Peter Pappas ${ }^{9}$
}

${ }^{1}$ Division of Infectious Diseases, Johns Hopkins University, Baltimore, MD, USA

${ }^{2}$ University of Pittsburgh Medical Center, Pittsburgh, PA, USA

${ }^{3}$ Centers for Disease Control and Prevention, Atlanta, GA, USA

${ }^{4}$ University of Michigan Medical School and Veterans Affairs Ann Arbor Healthcare System, Ann Arbor, MI, USA

${ }^{5}$ University of Pennsylvania, Philadelphia, PA, USA

${ }^{6}$ Washington University School of Medicine, St. Louis, MO, USA

${ }^{7}$ University of Toronto Medical Center, Toronto, ON, Canada

${ }^{8}$ University of Queensland, Brisbane, QLD, Australia

${ }^{9}$ University of Alabama at Birmingham, Birmingham, AL, USA

\section{Correspondence}

Robin K. Avery, Division of Infectious

Diseases, Johns Hopkins University,

Baltimore, MD, USA.

Email: ravery4@jhmi.edu

Funding information

Centers for Disease Control and Prevention, Grant/Award Number: Mycotic Diseases Branch

\begin{abstract}
Background: Most studies of post-transplant CMV infection have focused on either solid organ or hematopoietic cell transplant (HCT) recipients. A large prospective cohort study involving both lung and HCT recipients provided an opportunity to compare the epidemiology and outcomes of CMV infections in these 2 groups.

Methods: Patients were followed up for 30 months in a 6-center prospective cohort study. Data on demographics, CMV infections, tissue-invasive disease, recurrences, rejection, and immunosuppression were recorded.

Results: The overall incidence of CMV infection was 83/293 (28.3\%) in the lung transplant group and $154 / 444(34.7 \%)$ in the HCT group $(P=.0706)$. Tissue-invasive CMV disease occurred in 8/83 (9.6\%) of lung and 6/154 (3.9\%) of HCT recipients with CMV infection, respectively $(P=.087)$. Median time to $C M V$ infection was longer in the lung transplant group (236 vs 40 days, $P<.0001$ ), likely reflecting the effects of prophylaxis vs preemptive therapy. Total lgG levels of $<350 \mathrm{mg} / \mathrm{dL}$ in lung recipients and graft vs host disease (GVHD) in HCT recipients were associated with increased CMV risk. HCT recipients had a higher mean number of CMV episodes $(P=.008)$, although duration of viremia was not significantly different between the 2 groups. CMV infection was not associated with reduced overall survival in either group.

Conclusions: Current CMV prevention strategies have resulted in a low incidence of tissue-invasive disease in both lung transplant and $\mathrm{HCT}$, although $\mathrm{CMV}$ viremia is still relatively common. Differences between the lung and HCT groups in terms of time to $C M V$ and recurrences of $C M V$ viremia likely reflect differences in underlying host immunobiology and in CMV prevention strategies in the modern era.

KEYWORDS

cytomegalovirus, lung transplant, multicenter cohort study, stem cell transplant
\end{abstract}




\section{1 | INTRODUCTION}

Although there is extensive literature on CMV and CMV prevention in transplantation, ${ }^{1-5}$ most studies have focused on either solid organ transplantation or HCT alone, and have rarely compared these 2 groups in terms of CMV incidence, risk factors, virologic features, and outcomes. The Organ Transplant Infection Prevention (OTIP) study is a 6-center cohort study involving lung transplant and HCT recipients with parallel data collection methodologies. Although originally established for the purpose of investigating the epidemiology and environmental risk factors for post-transplant fungal infections, the OTIP study design has provided a unique opportunity for comparing and contrasting various other infections in these 2 groups. $^{6}$ The current study focuses specifically on CMV infections in the OTIP cohort.

\section{2 | METHODS}

\section{1 | General}

During 2006-11, 6 academic transplant centers (University of Pittsburgh, University of Alabama at Birmingham, University of Michigan, Washington University, University of Pennsylvania, and Cleveland Clinic) contributed patients (5 centers for lung transplant recipients and 4 centers for HCT recipients). All patients provided written informed consent, and Institutional Review Board approval was obtained at each center. Detailed questionnaires were administered to each patient at baseline. Clinical assessments were performed weekly during the transplant admission and subsequent hospitalizations. After discharge, patients were contacted by phone weekly for the first 12 weeks post-transplant, and monthly after that, with a total follow-up period of up to 30 months. Infectious syndromes were defined according to National Nosocomial Infections Surveillance System criteria. ${ }^{7}$ A uniform case report form and electronic data entry form developed by the Centers for Disease Control and Prevention (CDC) was used by all centers, and information was forwarded to a central data repository at CDC.

\section{2 | Definitions}

Definitions of tissue-invasive CMV disease (end-organ disease) and $\mathrm{CMV}$ viremia followed the standard definitions that were in use at the time of the study. ${ }^{8}$ Although subsequently updated definitions have been published, ${ }^{9}$ which should now replace the older definitions for clinical trials, the newer definitions were not yet published at the time this study was conducted. Therefore, "tissue-invasive CMV" indicated positive histopathology or a positive CMV immunostain on a tissue biopsy (except for CMV retinitis, which was diagnosed by ophthalmologic examination). "CMV viremia" was defined as any detection of CMV in blood by CMV quantitative polymerase chain reaction (CMV PCR) or pp65 antigenemia testing. For purposes of this study, data were not collected on the intermediate category of "CMV syndrome" which has been defined for solid organ transplant, but not HCT recipients, ${ }^{8,9}$ and in the current study, such patients were categorized as having CMV viremia rather than tissue-invasive disease, even if symptomatic. A CMV episode was considered resolved if the CMV PCR or antigenemia test was negative (undetectable) twice, on assays obtained at least 1 week apart, and a recurrence was defined as any CMV detection that occurred after at least 2 negative (undetectable) PCR or antigenemia test results had been obtained.

\section{3 | CMV detection assays}

CMV viral load monitoring was performed per each center's protocol. Most centers used CMV quantitative PCR assays during this time period, which was before the advent of the United States Food and Drug Administration (FDA)-approved PCR assay in IU/mL; therefore, CMV PCR assays were center-specific with results expressed as DNA copies/mL. A study core laboratory was not utilized in this study; therefore, CMV PCR measurements were not standardized among centers. ${ }^{10}$ One center used only pp65 antigenemia assays, and 2 centers used both pp65 antigenemia assays and CMV PCR assays. Shell vial cultures for CMV were performed on bronchoalveolar lavage fluid per center protocol. Duration of CMV prophylaxis and use of preemptive strategies are described in the Results section below.

\subsection{Statistics and data analysis}

Data collected by the 6 OTIP centers were transmitted to CDC via an electronic case report form. Data were collected in real time and forwarded at least monthly. Final data cleaning and statistical analysis were performed at the CDC. Chi-square and Fisher's exact tests, as appropriate, were used to compare categorical variables, and $t$ tests and Wilcoxon rank-sum tests were used to compare continuous variables. In all analyses, the level of significance was set at $\alpha=.05$. All analyses were done using SAS software (version 9.3, SAS Institute, Inc., Cary, NC).

\section{RESULTS}

\section{1 | Patients and pre-transplant characteristics}

Demographics and pre-transplant characteristics of the study patients are summarized in Tables 1 and 2. There were 293 lung transplant recipients from 5 centers (University of Pittsburgh 174, University of Michigan 43, University of Alabama 38, Cleveland Clinic 33, and University of Pennsylvania 5), and 444 HCT recipients from 4 centers (Washington University 207, University of Michigan 153, University of Pennsylvania 60, University of Alabama 24). The patients were not evenly distributed among centers; 1 transplant center (University of Pittsburgh) accounted for $59.4 \%$ of the lung transplant recipients, and 2 transplant centers (Washington University and University of Michigan) accounted for $46.6 \%$ and $34.5 \%$ of the HCT recipients, respectively. Transplant types, underlying diagnoses, pre-transplant conditioning and induction 
TABLE 1 Selected demographics, pre-transplant, and transplant features of lung transplant recipients with and without CMV infection

\begin{tabular}{|c|c|c|c|c|}
\hline & Total & CMV Infection - & CMV infection + & $P$ value \\
\hline Overall & 293 & 210 & 83 & \\
\hline Gender male (\%) & $168(57.3)$ & $121(57.6)$ & $47(56.6)$ & .8770 \\
\hline $\begin{array}{l}\text { Median age, years } \\
\text { (range) }\end{array}$ & $58.3(19.7-81.9)$ & $57.2(19.7-79.9)$ & $60.1(20.4-81.9)$ & .0869 \\
\hline Race Caucasian (\%) & 275 (93.9) & $195(92.9)$ & $80(96.4)$ & .4511 \\
\hline \multicolumn{5}{|c|}{ Underlying disease prompting transplant (\%) } \\
\hline Cystic fibrosis & $39(13.3)$ & $32(15.2)$ & $7(8.4)$ & \multirow[t]{3}{*}{.5393} \\
\hline COPD & $95(32.4)$ & $63(30.0)$ & $32(38.6)$ & \\
\hline $\begin{array}{l}\text { Idiopathic } \\
\text { pulmonary } \\
\text { fibrosis }\end{array}$ & $93(31.7)$ & 65 (31.0) & $28(33.7)$ & \\
\hline Type: Bilateral (\%) & $182(62.1)$ & $132(62.9)$ & $50(60.2)$ & .6674 \\
\hline Alemtuzumab (\%) & $173(59.0)$ & $130(61.9)$ & $43(51.8)$ & .1133 \\
\hline Basiliximab (\%) & $17(5.8)$ & $15(7.1)$ & $2(2.4)$ & .1662 \\
\hline Rejection (\%) & 211 (72.0) & $146(69.5)$ & 65 (78.3) & .1310 \\
\hline $\begin{array}{l}\operatorname{IgG}<350 \mathrm{mg} / \mathrm{dL}^{\mathrm{a}} \\
(\%)\end{array}$ & 17 (18.9) & 8 (13.1) & 9 (31.0) & .0424 \\
\hline $\mathrm{D}+/ \mathrm{R}-(\%)$ & $72(24.6)$ & $42(20)$ & $30(36.1)$ & .003 \\
\hline $\begin{array}{l}\text { Valganciclovir } \\
\text { prophylaxis (\%) }\end{array}$ & 267 (91.1) & $189(90.0)$ & $78(94.0)$ & .2809 \\
\hline
\end{tabular}

COPD, chronic obstructive pulmonary disease; IgG, immunoglobulin G.

${ }^{a}$ Checked in $30.1 \%$ of patients per clinician choice. Percentages refer to $\%$ of patients in whom IgG levels were checked. immunosuppression agents, CMV serostatus and prevention strategies are summarized in Tables 1 and 2.

In the lung transplant cohort, 72/293 (24.6\%) were in the highrisk CMV donor-seropositive, recipient-seronegative ( $D+/ R-)$ group. In the HCT cohort, 94/444 (21.2\%) were in the high-risk CMV donorseronegative, recipient-seropositive group ( $D-/ R+)$.

\section{2 | Overall incidence of CMV infection and risk factors for CMV}

The overall incidence of CMV infection was 83/293 (28.3\%) in the lung transplant group and $154 / 444$ (34.7\%) in the HCT group $(P=.0706)$. Tissue-invasive (end-organ) CMV disease occurred in 8 lung transplant recipients (9.6\% of those with CMV infection) and $6 \mathrm{HCT}$ recipients (3.9\% of those with CMV infection, $P=.8$.) Of 24 lung recipients with positive CMV shell vial BAL cultures, 7 (30\%) had CMV pneumonitis and 17 (70\%) did not (representing viral shedding without tissue-invasive disease.)

In the lung transplant group, none of the characteristics listed in Table 1 was significantly associated with risk for CMV. In the HCT group, $11 / 15$ (73.3\%) of African-American patients and 5/5 (100\%) of Asian patients developed CMV, as compared with 137/421 (32.7\%) of Caucasian patients, but given the small number of non-Caucasian patients, this should be considered a questionable finding. There was a difference among centers in terms of CMV incidence for the HCT group $(P=.0009)$, paralleling the percentages of unrelated donors at the 4 HCT centers (which ranged from 46\%-68\% unrelated donor HCT's), although a formal analysis of other factors contributing to center-specific differences was not performed. There were no center-specific differences in CMV incidence in lung transplant recipients. Mismatched-unrelated donor $\mathrm{HCT}$ recipients had the highest risk, in that $12 / 20(60 \%)$ of that group developed CMV infection, as compared with $57 / 177(32.2 \%)$ of matched-related donor and 85/245 (34.7\%) of matched-unrelated donor HCT recipients $(P=.066)$, but the number of mismatched-unrelated donor HCT's in this study was small (20/444 or $4.5 \%)$. There was no significant difference in $\mathrm{CMV}$ risk between $\mathrm{HCT}$ recipients who received myeloablative or nonmyeloablative regimens.

No peritransplant immunosuppressive regimens, including alemtuzumab induction, were significantly associated with CMV risk in lung transplant recipients. In the HCT group, the use of antithymocyte globulin (ATGAM) approached significance, in that 20/42 (47.6\%) of ATGAM-treated patients developed CMV $(P=.0642)$. Use of methotrexate for GVHD prophylaxis in HCT recipients was associated with a lower risk of CMV, with an odds ratio of $0.520(95 \% \mathrm{Cl}$, 0.313-0.864). None of the other immunosuppressive medications were associated with differential risk for CMV.

Rejection episodes occurred in $72 \%$ of lung transplant recipients, but rejection was not significantly associated with CMV in this group. GVHD (of any site or grade) occurred in $76.4 \%$ of HCT recipients overall, and occurrence of any GVHD was associated with CMV with an odds ratio of 1.627 (95\% Cl, 1.001-2.643).

Total immunoglobulin G (IgG) levels were checked (per clinician preference) in $30.7 \%$ of lung transplant recipients, and $18.9 \%$ 
TAB LE 2 Selected demographics, pre-transplant, and transplant features of hematopoietic cell transplant recipients with and without CMV infection

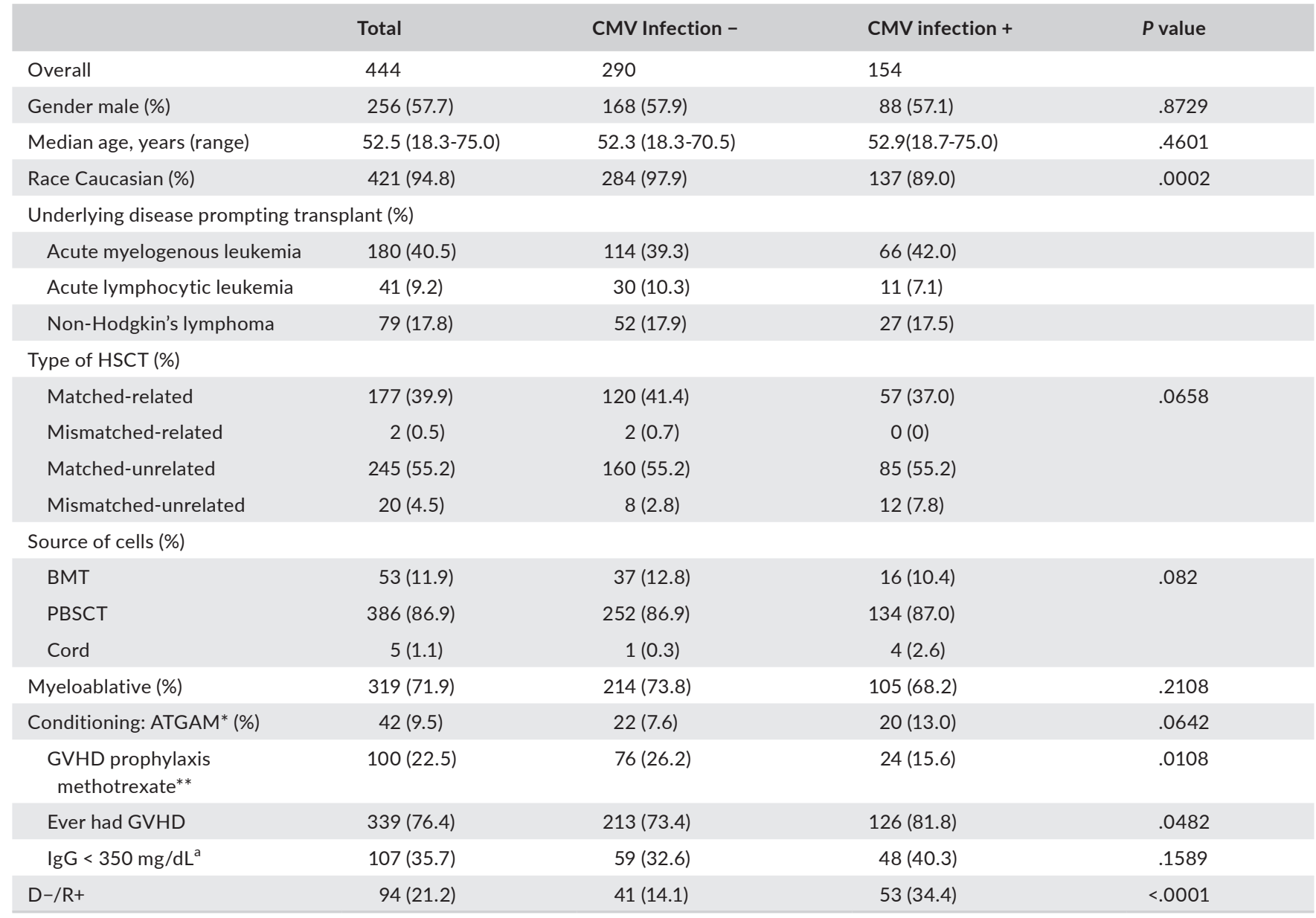

ATGAM, anti-thymocyte globulin; BMT, bone marrow transplant; GVHD, graft-vs-host disease; IgG, immunoglobulin G; PBSCT, peripheral blood stem cell transplant.

${ }^{a}$ Checked in $67.6 \%$ of patients per clinician choice. Percentages refer to \% of patients in whom IgG levels were checked.

${ }^{*} P$ values for all other conditioning regimen agents were not significant.

${ }^{* *} P$ values for all other GVHD prophylaxis agents were not significant.

of those who had IgG's checked had very low levels (<350 mg/dL). There was no association between CMV and IgG levels in lung recipients overall, but those with an IgG level of $<350 \mathrm{mg} / \mathrm{dL}$ had a higher incidence of CMV infection $(P=.0424)$. Total IgG levels were checked in 300/444 (67.6\%) of HCT recipients, and there was no association between IgG levels and CMV risk, despite $35.7 \%$ of those who had IgG checked having a low nadir lgG level $(<350 \mathrm{mg} / \mathrm{dL}$ ).

\subsection{Donor and recipient serostatus and CMV risk}

In the lung transplant group, the highest risk for developing CMV infection was in the donor-seropositive/recipient-seronegative ( $D+$ / R-) group, in which 30/72 developed CMV (41.7\%), followed by the $D+/ R+(33 / 90$, or $36.7 \%), D-/ R+(8 / 50$, or $16 \%)$, and $D-/ R-(2 / 49$, or $4.1 \%)$ groups. Twenty-eight donors and 13 recipients had missing serologic information.

In the HCT group, the highest risk for developing CMV infection was in the donor-seronegative, recipient-seropositive ( $D-/ R+)$ group, in which 53/94 (56.4\%) developed CMV, followed by the $\mathrm{D}+$ / $\mathrm{R}+(51 / 95$, or $53.4 \%), \mathrm{D}+\mathrm{R}-\mathrm{(5/39}$, or $12.8 \%)$, and D-/R- (19/157, or $12.1 \%)$ groups. Fifty-five donors and 26 recipients had missing serologic information.

\subsection{CMV prophylaxis duration, CMV incidence, and time to CMV}

CMV prophylaxis using valganciclovir was administered to $267 / 293$ (91.1\%) of lung recipients, and 48 (16.4\%) received IV ganciclovir as part of prophylaxis. The median length of CMV prophylaxis was 178 days (range, 1-977). Acyclovir was administered to 63 (21.5\%) and valacyclovir to $5(1.7 \%)$, generally to those who were CMV D-/ $\mathrm{R}-$, for prophylaxis of herpes simplex virus (HSV) and varicella-zoster virus (VZV). Only 7 (2.4\%) of lung transplant recipients received CMV immune globulin (CMVIg) for any indication.

In contrast, HCT recipients in general did not receive prophylaxis against CMV, but instead were managed with a strategy of serial 
TAB LE 3 Comparison of CMV between lung transplant and HCT recipients

\begin{tabular}{lccr} 
& Lung & HCT & P value \\
\hline Overall (n, \%) & $83(28.3)$ & $154(34.7)$ & .0706 \\
\hline $\begin{array}{l}\text { Median time to } \\
\text { CMV, days (range) }\end{array}$ & $236(4-689)$ & $40(4-666)$ & $<.0001$ \\
\hline $\begin{array}{c}\text { CMV recurrence (n, } \\
\%)\end{array}$ & $18 / 83(21.7)$ & $65 / 154(42.2)$ & .0015 \\
$\begin{array}{l}\text { Mean No. of CMV } \\
\text { episodes (range) }\end{array}$ & $1.34(1-4)$ & $1.63(1-5)$ & .0076 \\
\hline $\begin{array}{l}\text { Median duration of } \\
\text { viremia, days } \\
\text { (range) }\end{array}$ & $46.5(1-405)$ & $41(1-900)$ & .5612 \\
\hline
\end{tabular}

${ }^{\mathrm{a}} \mathrm{N}=166$ patients with information available.

monitoring of CMV PCR or pp65 antigenemia assays usually through Day 100 post-transplant, and preemptive therapy when the CMV PCR or antigenemia assay turned positive. HCT recipients generally received prophylaxis for HSV and VZV in the form of acyclo$\operatorname{vir}(315 / 444$, or $71 \%)$ or valacyclovir (183/444, or $41.2 \%)$ per their centers' protocols, while undergoing CMV monitoring. In the HCT group, 24 patients received CMVIg, but 23 of these had CMV infection (hence, CMVIg was not used for prophylaxis).

Time to first detection of CMV was significantly different between lung transplant recipients with a median of 236 days (range, 4-689) and HCT recipients with a median of 40 days (range, 4-666; $P<.0001$ ) (Table 3). Twelve HCT patients (7.7\%) developed CMV infection prior to engraftment. In the lung transplant group, $20 \mathrm{pa}$ tients (27\% of those with CMV infection) developed their first CMV episode while still on anti-CMV prophylaxis.

\subsection{CMV clinical and virologic features}

Tissue-invasive CMV disease (end-organ disease) was uncommon in this cohort, occurring in $8 / 83$ (9.6\%) of lung recipients with CMV, or $8 / 293(2.7 \%)$ of all lung recipients, and 6/155 (3.9\%) of HCT recipients with CMV, or $6 / 444(1.4 \%)$ of all HCT recipients $(P=.087)$. Sites of end-organ involvement in lung recipients included CMV pneumonitis in 7, CMV colitis in 2, and other sites in 2, whereas sites in HCT recipients included CMV enterocolitis in 4, CMV pneumonitis in 1, and CMV hepatitis in 1.

Recurrences of CMV occurred in 18/83 (21.7\%) of lung transplant recipients and 65/154 (42.2\%) of HCT recipients ( $P=.0016)$. The mean number of CMV episodes was significantly higher in HCT recipients, with $1.63 \mathrm{CMV}$ episodes per patient (range, 1-5 episodes) as compared with lung transplant recipients, who had a mean of 1.34 CMV episodes per patient (range, 1-4 episodes, $P=.0076$ ). Information on duration of viremia was available for 166/237 (70\%) of the patients with CMV episodes, and the median duration was 46.5 days for lung transplant recipients (range, 1-405 days) vs 41 days for HCT recipients (range, 1-900 days), which was not significantly different $(P=.5612)$.
Fungal infections occurred in 24 lung transplant recipients (8.2\%) and $48 \mathrm{HCT}$ recipients (10.8\%), with no significant differences in fungal infection incidence between the groups who did and did not develop CMV infection.

Antiviral agents other than ganciclovir and acyclovir derivatives were administered to relatively few lung recipients (6 foscarnet, 2 cidofovir, all of whom were in the group that developed CMV infection) and relatively more HCT recipients (40 foscarnet, 29 cidofovir). The specific reasons for choice of antiviral agent were not recorded in this dataset. Among HCT recipients, 8 foscarnet and 11 cidofovir recipients were in the group that never developed CMV viremia, so these antiviral agents may have been administered for treatment of other viruses (eg, HSV, VZV, HHV-6, BKV, or adenovirus infection). In addition, it is possible that some foscarnet use in the HCT group with CMV (32 patients) might have reflected clinicians' desire to avoid the hematologic toxicities of ganciclovir/valganciclovir in patients with pre-engraftment $\mathrm{CMV}$ or borderline neutropenia.

\section{6 | Survival}

Overall survival in this cohort at 6,12 , and 18 months was $89.4 \%$, $82.9 \%$, and $80.6 \%$ for lung transplant recipients, and $72.3 \%, 59.2 \%$, and $54.3 \%$ for HCT recipients. There was no significant reduction in survival in either cohort between those who developed CMV infection and those who did not.

\section{4 | DISCUSSION}

The results of this large multicenter cohort study highlight both the differences in host immunobiology and the effects of different antiviral prevention strategies between lung transplant and $\mathrm{HCT}$ recipients. This study demonstrates that CMV viremia remains common in both types of transplant recipients in the modern era, although tissue-invasive CMV disease has become uncommon with the use of current CMV prevention strategies. ${ }^{1-5}$ Since HCT programs rely on preemptive monitoring rather than universal antiviral prophylaxis, ${ }^{4,5}$ it is not surprising that $\mathrm{CMV}$ viremia remains common in this group, but it also occurred in $28.3 \%$ of lung recipients despite prophylaxis. Thus, it appears that the success of CMV prevention in both groups has not been in the complete suppression of viremia, but rather in the prevention of highly symptomatic CMV and end-organ CMV disease.

Recurrences of viremia were more common in $\mathrm{HCT}$ recipients than in lung transplant recipients, likely reflecting host factors and the requirement for HCT recipients to reconstitute their immune system from the donor and concomitantly to develop CMV-specific immune function.

This study confirmed a number of CMV risk factors that have been described in previous literature, including the high-risk status of the donor-seropositive, recipient-seronegative ( $D+/ R-)$ group in solid organ transplant recipients and the donor-seronegative, recipient-seropositive $(D-/ R+)$ group in $\mathrm{HCT}^{1-5}$ The former reflects 
the de novo acquisition of donor-transmitted CMV infection in a solid organ transplant recipient without antecedent CMV-specific immunity, whereas the latter reflects the reconstitution of a donor immune system without antecedent CMV-specific immunity in the context of a recipient at risk for reactivation of pre-existing latent $\mathrm{CMV}$ infection. This study also confirms that the risk of CMV viremia varies with the type of $\mathrm{HCT},{ }^{11}$ for example higher risk in mismatchedunrelated HCT recipients, although these constituted only $4.5 \%$ of HCT recipients in this study. Cord transplant recipients constituted only a small fraction of patients in the current study, precluding meaningful comparisons.

Of note, although the number of patients with tissue-invasive $\mathrm{CMV}$ disease in this cohort was small, it is interesting to note that the sites of end-organ involvement showed a predominance of CMV pneumonitis in lung recipients, and gastrointestinal CMV in HCT recipients. Lung recipients have traditionally been noted to be at risk for CMV pneumonitis, given the propensity for CMV invasive disease to localize to the allograft. However, the finding that approximately $2 / 3$ of lung recipients with positive CMV shell vial cultures from BAL did not have documented CMV pneumonitis parallels that of the study of BAL CMV viral loads by Chemaly et al, in which detection of CMV without tissue invasion occurred in about $2 / 3$ of those with positive BAL CMV cultures, and this shedding was associated with lower levels of quantitative BAL viral load. Therefore, a positive CMV shell vial culture from BAL does not necessarily indicate CMV pneumonitis. ${ }^{12}$

One of the original goals of this study was the comparison of infection outcomes between centers that do or do not use alemtuzumab induction in lung transplantation ${ }^{13}$; we found that there was no significant difference in CMV infection in relation to alemtuzumab use in this cohort. Of the immunosuppressive agents administered, the only significant association was a lesser risk of CMV in HCT recipients who received methotrexate for GVHD prophylaxis. There was a trend toward increased $\mathrm{CMV}$ incidence in $\mathrm{HCT}$ recipients who received antilymphocyte therapy. Other immunosuppressive agents and acute rejection in lung recipients were not associated with differential CMV risk in this study. There may still be inherent increased risk associated with certain immunosuppressive regimens and with acute rejection, but this risk might have been compensated for by heightened awareness and interventions on the part of clinicians.

Hypogammaglobulinemia was common in both groups, but was not associated with CMV risk in this study, except in lung recipients in the lowest IgG group ( $<350 \mathrm{mg} / \mathrm{dL}$ ). A previous study of hypogammaglobulinemia in lung transplantation had identified low IgG levels as associated with increased risk for tissue-invasive CMV disease, although not for CMV viremia in general. ${ }^{14}$ On the other hand, any occurrence of GVHD in this study was associated with increased CMV risk in HCT recipients.

The effects of different CMV prevention strategies were evident in the time to CMV data. The standard of CMV prevention in lung transplantation has traditionally been prophylaxis using IV ganciclovir and more recently valganciclovir. The randomized trial of $3 \mathrm{vs}$ 12 months valganciclovir prophylaxis by Palmer et al, ${ }^{15}$ published when the current study was nearly completed, demonstrated the benefits of longer durations of prophylaxis in lung transplant recipients. Before that, there was already a trend toward longer prophylaxis in lung transplant programs, as seen in previous nonrandomized trials. ${ }^{16,17}$ The median prophylaxis duration of almost 6 months for lung transplant recipients in the current study was thus reflective of a shift in practice occurring around that time. Therefore, it is not surprising that the initial detection of CMV viremia occurred much later in lung transplant recipients ("late CMV" after completion of prophylaxis), ${ }^{18}$ as compared with $\mathrm{HCT}$ recipients who received preemptive monitoring.

CMV infection and its treatment may predispose to fungal infections, either through cytopenias or by immunosuppressive effects of $\mathrm{CMV}$ itself, but we observed no significant difference in frequency of invasive fungal infections in patients who did and did not develop CMV in this study.

A comparison with incidence of CMV disease in other studies, and with historical incidence of CMV disease in previous eras, is illuminating. In the early years of lung transplantation, there was a high incidence of CMV pneumonitis and a high mortality, particularly in CMV primary infection. For example, in the pre-prophylaxis era, the incidence of CMV infection was $80 \%$, and CMV disease occurred in $31 \%$ of all lung recipients. ${ }^{19}$ In 1 study, prophylaxis using a delayed-ganciclovir regimen reduced these numbers to $48 \%$ and 10\%, respectively. ${ }^{19}$ The VAL038 study, ${ }^{15}$ a randomized controlled trial which compared 3 vs 12 months of valganciclovir prophylaxis in lung transplant recipients, reported a $32 \%$ incidence of CMV disease in the short-course prophylaxis group. The numbers in our current study are closer to those of the long-course prophylaxis group in the VAL 038 study (which had a $4 \%$ incidence of CMV disease).

In the HCT realm, much of our knowledge comes from the large studies performed at the Fred Hutchinson Cancer Research Center (FHCRC). For example, Green et $\mathrm{al}^{4}$ reported an incidence of CMV disease of $5.2 \%$ in those monitored with CMV PCR, and $5.8 \%$ in those monitored with antigenemia assays. In a separate analysis from FHCRC, Erard et $\mathrm{al}^{5}$ noted an improvement in mortality caused by CMV pneumonitis in HCT over time. Randomized clinical trials can also provide insight into background incidence. For example, in the placebo group of the Maribavir HCT prophylaxis study, CMV disease occurred in 5\% and CMV infection occurred in 30\%-37\%, depending on assay and excluding D-/R-. ${ }^{20}$ This correlates quite closely with the incidence of CMV infection of $34.7 \%$ in the HCT cohort in our study, although the incidence of tissue-invasive disease was somewhat lower in our study, being only $1.4 \%$ of total HCT recipients (3.9\% of those who had CMV viremia). These differences may reflect center-specific protocols for conditioning and GVHD prophylaxis, different case mixes and risk profiles of the recipients, different applications of standard definitions, or other factors. However, in all 3 of the above studies, tissue-invasive disease incidence was relatively low in the current era compared with the historical rates from the earlier years of BMT, in which as many as $43 \%$ developed CMV disease, frequently with $C M V$ pneumonitis which was associated with a high mortality. ${ }^{21}$ Somewhat surprisingly, we observed no association 
between CMV infection and reduction of overall survival in the current study. It is possible that other aspects in the modern era of CMV management, such as modulation of immunosuppression during and after viremia episodes, could theoretically have counterbalanced an adverse impact of CMV on survival.

\section{5 | LIMITATIONS}

This study had several limitations. Patients were not evenly distributed among centers, with 1 center contributing over half of the lung transplant recipients, and 2 centers contributing the majority of the HCT recipients. This was a predominantly Caucasian cohort across all 6 centers, and results may not be generalizable to other racial/ethnic groups. Some CMV serologies were missing, particularly in HCT donors. Centers varied in their use of induction and maintenance immunosuppression and antimicrobial prophylaxis. Center-specific protocols were used for CMV prevention, and for monitoring of CMV viremia; the duration and frequency of monitoring varied, depending on center practices and risk stratification. To obtain a truly accurate incidence of CMV viremia, centers would have had to adhere to a uniform monitoring protocol; therefore, the incidence of viremia should be interpreted with this caveat in mind. The CMV PCR assays used during this time period differed among centers, as this study was performed before the introduction of the FDA-approved CMV PCR assay, that is now internationally accepted. One center used only pp65 antigenemia assays, and 2 centers used both pp65 antigenemia and PCR over time. It is likely that significant inter-laboratory variation existed among the centers using quantitative CMV PCR assays ${ }^{9}$ and therefore, planned comparisons of CMV median and peak viral loads were eliminated from the data analysis plans. Thresholds for initiating CMV therapy varied from center to center and within centers over time, reflecting changes in assays and in clinical practice. The reasons for use of foscarnet and cidofovir were not recorded, including resistance genotype testing, therefore precluding observations about antiviral-resistant CMV. The lack of data collection on the intermediate category of "CMV syndrome" in lung transplant recipients may have underestimated the severity of symptomatic disease in this group. Finally, the study was not designed to assess the impact of infections on bronchiolitis obliterans syndrome or chronic lung allograft dysfunction in lung recipients or on graft survival/graft loss in HCT recipients, although those issues are of great interest to clinicians.

\section{6 | CONCLUSIONS}

Despite the limitations noted above, interesting comparisons have emerged between multicenter cohorts of lung transplant and HCT recipients in the modern era, with regards to $\mathrm{CMV}$ incidence, risk factors, clinical and virologic characteristics, and patient outcomes. These likely reflect both the disparate nature of host responses to
CMV, and also prevention strategies that are differentially applied to these groups because of the high risk of cytopenias in HCT recipients from prolonged use of valganciclovir and ganciclovir. Evidencedriven prophylaxis duration has become increasingly longer in lung transplant recipients, but preemptive monitoring and directed therapy remain the standard in HCT recipients. In both groups, the success of prevention strategies is reflected in an overall low incidence of tissue-invasive disease (despite a continued incidence of viremia) and the lack of a detrimental impact of CMV viremia on survival in both groups. Other findings include a significantly earlier onset of CMV viremia in HCT recipients, likely related to the use of preemptive therapy rather than prophylaxis, and also more CMV recurrences in the HCT group. The increased use of antivirals other than ganciclovir/valganciclovir in the HCT group with CMV may reflect the concerns of HCT clinicians regarding the risk of neutropenia from ganciclovir derivatives. Taken together, these results enable us to understand how far the field of CMV prevention has come, and also to provide historical background for assessments of the impact of new immunosuppressive and antiviral agents that may be introduced in the future.

\section{AUTHOR CONTRIBUTIONS}

Study Design: All authors; Data Analysis: KB, AAC; Data Interpretation: All authors; Drafting of the Manuscript: RKA; Critical Review and Revision of the Manuscript: All authors.

\section{ORCID}

Robin K. Avery (iD) http://orcid.org/0000-0001-7692-3619

Erik R. Dubberke iD http://orcid.org/0000-0003-3779-2796

\section{REFERENCES}

1. Razonable RR, Humar A, and the AST ID Community of Practice. Cytomegalovirus in solid organ transplantation. Am J Transplant. 2013;13:93-106.

2. Kotton CN, Kumar D, Caliendo AM, et al. International consensus guidelines on the management of cytomegalovirus in solid organ transplantation. Transplantation. 2010;89:779-795.

3. Snydman DR, Limaye AP, Potena L, Zamora MR. Update and review: state-of-the-art management of cytomegalovirus infection and disease following thoracic organ transplantation. Transplant Proc. 2011;43(3 Suppl):S1-S17.

4. Green ML, Leisenring W, Stachel D, et al. Efficacy of a viral load-based, risk-adapted, pre-emptive treatment strategy for prevention of cytomegalovirus disease after hematopoietic cell transplantation. Biol Blood Marrow Transplant. 2012;18: 1687-1699.

5. Erard V, Guthrie KA, Seo S, et al. Reduced mortality of cytomegalovirus pneumonia after hematopoietic cell transplantation due to antiviral therapy and changes in transplantation practices. Clin Infect Dis. 2015;61:31-39.

6. Schuster MG, Cleveland AA, Dubberke ER, et al. Infections in hematopoietic cell transplant recipients: results from the organ transplant infection project, a multicenter, prospective cohort study. Open Forum Infect Dis 2017;4:ofx050. 
7. Garner JS, Jarvis WR, Emori TG, et al. CDC definitions for nosocomial infections, 1988. Am J Infect Control. 1988;16:128-140.

8. Ljungman P, Griffiths P, Paya C. Definitions of cytomegalovirus infection and disease in transplant recipients. Clin Infect Dis. 2002;34:1094-1097.

9. Ljungman $\mathrm{P}$, Boeckh M, Hirsch $\mathrm{HH}$, et al. Definitions of cytomegalovirus infection and disease in transplant patients for use in clinical trials. Clin Infect Dis. 2017;64:87-91.

10. Pang XL, Fox JD, Fenton JM, et al. Interlaboratory comparison of cytomegalovirus viral load assays. Am J Transplant. 2009;9: 258-268.

11. Goldsmith SR, Slade M, DiPersio JF, et al. Cytomegalovirus viremia, disease, and impact on relapse in T-cell replete peripheral blood haploidentical hematopoietic stem cell transplantation with post-transplant cyclophosphamide. Haematologica. 2016;101:e465-e468. Epub 2016 Jul 21.

12. Chemaly RF, Yen-Lieberman B, Chapman J, et al. Clinical utility of cytomegalovirus viral load in bronchoalveolar lavage in lung transplant recipients. Am J Transplant. 2005;5:544-548.

13. Peleg AY, Husain S, Kwak EJ, et al. Opportunistic infections in 547 organ transplant recipients receiving alemtuzumab, a humanized monoclonal CD52 antibody. Clin Infect Dis. 2007;44 204-212.

14. GoldfarbNS,AveryRK,GoormasticM,etal.Hypogammaglobulinemia in lung transplant recipients. Transplantation. 2001;71:6.

15. Palmer SM, Limaye AP, Banks M, et al. Extended valganciclovir prophylaxis to prevent cytomegalovirus after lung transplantation: a randomized, controlled trial. Ann Intern Med. 2010;152:761-769.

16. Zamora MR, Nicolls MR, Hodges TN, et al. Following universal prophylaxis with ganciclovir and cytomegalovirus immune globulin, valganciclovir is safe and effective for prophylaxis of CMV following lung transplantation. Am J Transplant. 2004;4:1635-1642.

17. Valentine VG, Weill D, Gupta MR, et al. Ganciclovir for cytomegalovirus: a call for indefinite prophylaxis in lung transplantation. J Heart Lung Transplant. 2008;27:875-881.

18. Sun HY, Wagener MM, Singh N. Prevention of posttransplant cytomegalovirus disease and related outcomes with valganciclovir: a systematic review. Am J Transplant. 2008;8:2111-2118.

19. Brumble LM, Milstone AP, Loyd JE, et al. Prevention of cytomegalovirus infection and disease after lung transplantation: results using a unique regimen employing delayed ganciclovir. Chest. 2002;121:407-414.

20. Marty FM, Ljungman P, Papanicolaou GA, et al. Maribavir prophylaxis for prevention of cytomegalovirus disease in recipients of allogeneic stem-cell transplants; a phase 3, double-blind, placebocontrolled, randomised trial. Lancet Inf Dis. 2011;11:284-292.

21. Goodrich JM, Mori M, Gleaves CA, et al. Early treatment with ganciclovir to prevent cytomegalovirus disease after allogeneic bone marrow transplantation. N Eng J Med. 1991;325:1601-1607.

How to cite this article: Avery RK, Silveira FP, Benedict K, et al. Cytomegalovirus infections in lung and hematopoietic cell transplant recipients in the Organ Transplant Infection Prevention and Detection Study: A multi-year, multicenter prospective cohort study. Transpl Infect Dis. 2018;20:e12877. https://doi.org/10.1111/tid.12877 